\title{
ON THE DETECTION OF BINARY Be STARS
}

\author{
R. S. POLIDAN \\ Dept. of Astronomy, University of California, Los Angeles, Calif., U.S.A.
}

\begin{abstract}
Possible methods for detecting the presence of a cool companion to a Be star are discussed. Photometric observations are shown to be incapable of detecting companions in all but the most extreme cases. Spectroscopic investigation is also unlikely to yield many new discoveries. It, however, remains the most promising method for the detection of binary Be stars. The four known binary Be stars are also discussed.

Infrared calcium triplet emission in Be stars is discussed in detail. The lines are shown to originate in a region of large optical thickness and low temperature $(T \sim 5000 \mathrm{~K})$. The possible connection between the presence of calcium triplet emission and binary nature is briefly discussed.
\end{abstract}

\section{Introduction}

If one wishes to discuss the possibility that some Be stars are interacting binary stars it becomes necessary to define in as exact terms as possible the physical properties of the system. As pointed out by Dr Harmanec, mass transfer calculations have not yielded a unique model for Be binary stars. It is possible to obtain a wide variety of systems by simply changing the starting conditions or the stage of mass transfer in which the binary is viewed. There is, however, a specific type of system which we at Los Angeles feel is representative of many of the Be binary stars. This is a system that bears a close resemblance to the semi-detached eclipsing systems collectively known as Algols. In the case of Be stars the brighter component (primary) is an early-type (B0 to A0) main-sequence band object surrounded by an extensive ring of gas. The cooler companion (secondary) is a star of spectral type $\mathrm{G}$ or $\mathrm{K}$ (possibly M) and of luminosity class III or IV (in some cases II). The physical size of the system must be larger than that of a typical Algol system in order to accommodate the large disk of gas. Periods greater than 10 to 20 days will satisfy this requirement. An upper limit to the period will be set by the condition that mass transfer must take place; this in principle can be 1000 days or more. However, periods in excess of a few hundred days are expected to be rare. Regarding masses, the secondary is expected to show evidence of mass loss: a very low mass for its position in the HR diagram. The primary would be expected to have a mass typical for its location in the HR diagram. It also may have a slight overluminosity due to the accretion of mass from the disk; this is expected to be significant only in the early stages of mass transfer. Typically, mass ratios in the range $5: 1$ to $15: 1$ in favor of the primary are expected.

This definition of a Be binary star still has a considerable range of physical parameters. It does, however, allow discussion of some of the observable properties of these systems. In particular it allows the question of detectability to be discussed.

\section{Detectability}

The question of the detectability of a companion to a Be star must be answered on many fronts. A companion can be detected through photometric anomalies in the $\mathrm{Be}$ 
star (eclipses or a composite flux distribution), radial velocity variations indicative of orbital motion, periodic activity in the spectrum of the star, and perhaps a few others. Since we are considering a hot star combined with a cooler larger star photometric detection would seem to be the most promising way to search for a secondary. In particular the recent advances in far infrared $(\lambda>2 \mu)$ photometry should aid in the detection of the red component. The presence of infrared free-free emission in $\mathrm{Be}$ stars should reduce the probability of detection, in some cases making impossible the detection of the companion by photometric means. If we look at the Be stars with known companions to resolve this question we find a surprising and somewhat unsettling answer. Allen (1973) has obtained infrared $(H K L)$ observations of the Be binaries for which we detect the presence of a companion in the near infrared. If we look at the stars with M-type companions, 17 Lep (Slettebak, 1951; Cowley, 1968) and XX Oph (Lockwood et al., 1975), we find that the companion is photometrically visible. Both are classified by Allen as having infrared colors similar to those of late type (i.e. M) stars. Allen has also observed the star BD+14\%387. An infrared spectrum obtained of this object this summer shows a spectrum very similar to that of $\mathrm{XX}$ Oph, possibly even to the faint M-star absorption spectrum that is seen in XX Oph. Allen also photometrically classifies this star as having infrared colors similar to that of an M-type star. Thus, it would seem that detection of M-type companions is possible from infrared photometric observations. However, the model for the binary Be star suggested above states that a G- or K-type star is a much more likely secondary component than an M-type star. Fortunately, Allen has obtained HKL colors of the three Be stars known to contain $\mathrm{G}$ or $\mathrm{K}$-type companions: AX Mon, HD 218393, and HR 894 (HD 18552). All three were found to show no evidence of a companion; the infrared colors could be represented by an early-type continuum plus emission from an optically thin ionized hydrogen envelope. This is in spite of the fact that at $\lambda 8500$ the companions contribute respectively $65 \%, 40 \%$, and $25 \%$ of the total light of the system. This pronounced invisibility of the K-type companion becomes even more apparent if one looks not at a classical Be star (though the star does show very weak emission at $\mathrm{H} \alpha$ ) but at the eclipsing binary star $\beta$ Per (Algol). This may appear to be an unfair comparison, but using the results of Hill and Hutchings (1970) for the three components of Algol it is found that the B-type star contributes only $60 \%$ of the total flux at any point beyond approximately $2 \mu$. Of the remaining flux, $10 \%$ is contributed by the A-type component and $30 \%$, almost $\frac{1}{3}$, is contributed by the $\mathrm{K}$-type subgiant. This binary is then quite representative of what is expected for a Be binary star. Algol also has high quality photometric observations available from $0.36 \mu(U)$ through $2.2 \mu(K)$ (Johnson et al., 1966) and from $2.3 \mu$ to $11.4 \mu$ (Gerhz et al., 1975). Gerhz et al. used Algol as a representative normal B8V star in their $2.3 \mu$ to $19.5 \mu$ photometric study of Be stars. They also observed the truly normal unreddened B8V star $\beta$ Lib as did Johnson et al. A comparison of these two B8V stars gives a surprising and as yet not completely understood result. NO significant difference is found between the flux distributions of $\beta$ Per and $\beta$ Lib that cannot be accounted for by reddening $(E(B-V)$ for $\beta$ Per is 0.04$)$. The $2.3 \mu$ to $11.5 \mu$ distributions are significantly different only at the $5 \mu$ point. What is most surprising is that the 8 -color observations of Johnson et al. reveal no infrared excess, even through the K-type secondary contributes fully $30 \%$ of the total light at the $K(2.2 \mu)$ magnitude. 
The results of Gerhz et al. should not be entirely unexpected. In the region beyond 2 microns all three components are on the Rayleigh-Jeans portion of their energy distributions. Hence, the distribution depends only on the ratio of their temperatures and is, therefore, independent of wavelength. This is the primary reason for the failure of far infrared photometry to detect the companions in AX Mon, HD 218393, HR 894, and in $\beta$ Per. Far infrared photometric observations cannot distinguish differences in the distributions of flux of stars hotter than approximately $3600 \mathrm{~K}$.

The results of the observations of Johnson et al. are much more difficult to understand. From the parameters obtained by the solution of the light curve of Algol (Hill and Hutchings, 1970) a $V-K$ excess in excess of $0.4 \mathrm{mag}$. is expected. The observed $V-K$ excess is only $0.13 \mathrm{mag}$., very nearly what is expected from reddening and the observed $B-V$ excess of Algol. The reason for this lack of excess is unknown. It is unlikely that the cause is uncertainty in the physical parameters, radii and temperatures, of the components of Algol. More likely is our lack of knowledge of the effects of mass loss or the proximity of the critical Roche surface on a stellar atmosphere.

The conclusion that one is forced to accept based on the above observed results is that M-type companions to Be stars should be easily detected photometrically, but the expected most common type of secondary, a G or K-type star, is photometrically invisible.

One other photometric method of detection should at this point be mentioned: eclipses. If we have binary Be stars, then we should observe some of them to undergo eclipses. The question is one of what is the probability of an eclipse occurring in such a wide system and, with only scattered observations, what is the probability that the eclipse will be detected. It must be mentioned that many eclipsing ' $\mathrm{Be}$ ' stars do exist: they are usually called Algol-type eclipsing binary stars. As Plavec pointed out in his introductory address to this Symposium many Algol systems have emission spectra quite similar to those found in traditional non-eclipsing $\mathrm{Be}$ stars. The extensive spectroscopic observations that exist for Be stars do not, in general, exist for eclipsing binary stars. Therefore, little can be said about any other similarities between these two groups other than general appearance. Similarly Be stars generally do not have the extensive photometric coverage that many binary stars have. Certainly, none of the brightest Be stars could undergo an eclipse without being detected, but for fainter Be stars, objects for which observations are infrequent at best, eclipses could possibly be completely missed. The problem of eclipse probability and detection probability will be discussed in detail by Plavec later in this session.

Turning now to the spectroscopic methods for the detection of companions to Be stars one first thinks to look for radial velocity variations caused by orbital motion. Unfortunately, the low expected semi-amplitude (less than $10 \mathrm{~km} \mathrm{~s}^{-1}$ in many cases), the poor, rotationally broadened lines, and the possible contamination of the line by emission or absorption from the envelope make this method unlikely to yield many unchallenged discoveries. It can, however, be very helpful in finding the shorter period, 10 to 20 day, Be binary systems. If the eclipsing binary AU Mon was inclined to our line of sight such that it would not eclipse, it would appear simply as a B5 emission line star that underwent periodic radial velocity variations with a period of approximately 11 days. This would probably be the only way that a system similar to this could be identified as a binary star. 
Another means of discerning the binary nature of a Be star is through the detection of short term (days to months) periodic spectral changes possibly associated with the period of the system. Cowley has shown that AX Mon (Cowley, 1964) and 17 Lep (Cowley, 1968) display a mildly periodic activity associated with the orbital period. Peton (1974) in his exhaustive study of AX Mon established a definite periodic activity associated with the orbital period. He also discovered shorter and longer term variations associated with changes in the structure of the envelope. Doazan and Peton (1970) observed periodic velocity variations of the shell lines and profile variations of the $H \beta$ line in the Be binary star HD 218393. Peters (1972) discovered periodic spectral changes in the Be star HR 2142. These systems, in particular HR 2142, will be discussed along with other stars showing periodic or quasi-periodic spectral variations by Peters in the next paper in this session.

One other method for detecting spectroscopically the presence of a compansion is through the discovery of spectral lines incompatible with the photospheric and envelope spectrum of the Be star. Since the companion is expected to be considerably cooler than the B-type star we would, ideally, like to look as far into the red as possible. Unfortunately, a good low noise detector is required to see the weak lines. This limits us to regions shortward of approximately one micron. Because of the distribution of energy in a late-G early-K-type star, regions shorter than roughly $\mathrm{H} \alpha$ are unlikely to yield many discoveries. Thus, the current optimum spectral region for searching for companions to Be stars appears to be between $\lambda 7000 \AA$ and $\lambda 10000 \AA$.

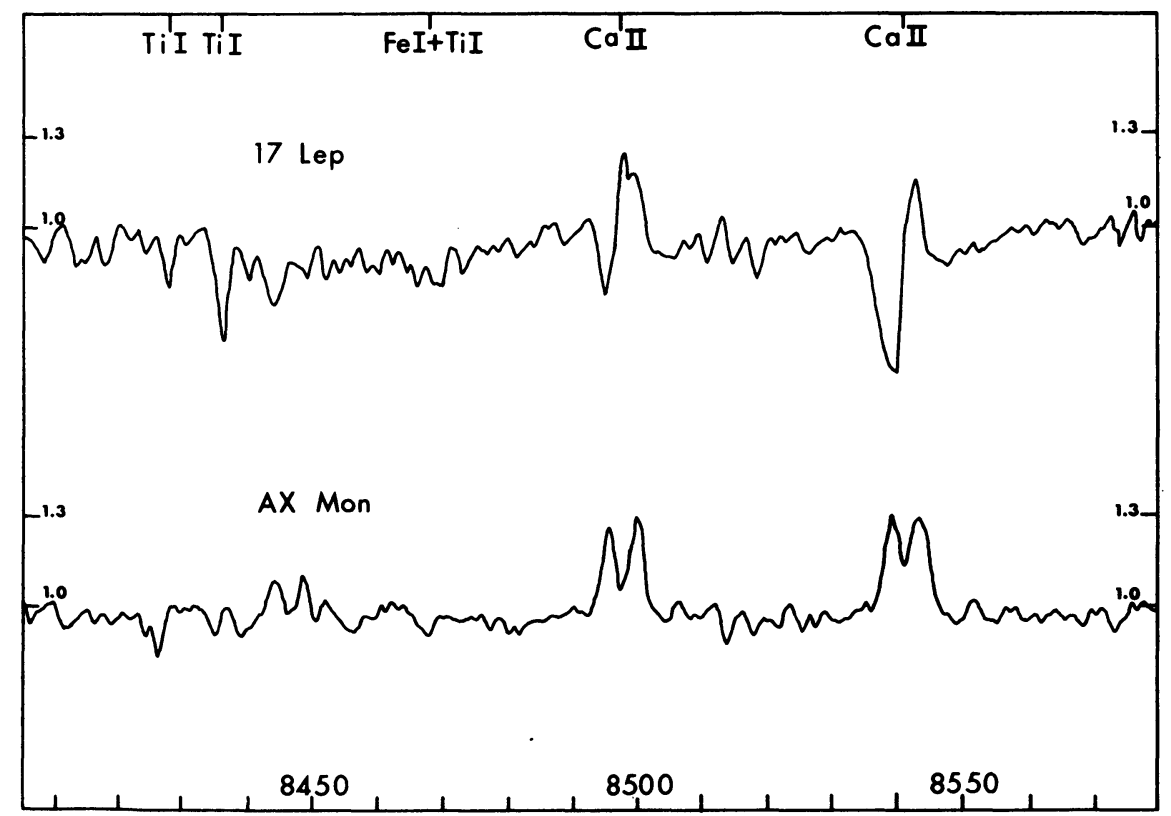

Fig. 1. A portion of the near infrared spectra of AX Mon and 17 Lep. The most prominent lines of the companions are marked. Ordinate is in units of intensity normalized to the continuum. Original dispersion: $23 \AA \mathrm{mm}^{-1}$. 
The most likely candidate lines to search for would be lines of $\mathrm{CN}$. They are strong in late type stars and cannot arise either in the photospheric or envelope spectrum of the Be star. The strongest band of $\mathrm{CN}$ has its head at $\lambda 10900 \AA$. This is, however, very near the center of the atmospheric $\Phi$ band of water. A weaker band with its head at $\lambda 9150 \AA$ is also contaminated by atmospheric water but less severely than the $\lambda 10900 \AA$ band. A third, even weaker, band, actually a pair of bands, exists near $\lambda 8200 \AA$. This too is badly contaminated by atmospheric water.

Strong atomic lines are not uncommion in the infrared spectra of late type stars. The infrared calcium triplet at $\lambda \lambda 8498,8542$, and $8662 \AA$ is particularly strong. The region surrounding the triplet also contains many weaker lines of $\mathrm{Fe}_{\mathrm{I}}$ and $\mathrm{Ti}$, lines that are not likely to be found in the envelope spectrum of the Be star. This region is also relatively free of photospheric features, except for the Paschen lines, and of shell features (Paschen lines, O I $\lambda 8446 \AA, N_{I} \lambda 8680 \AA$, and in very rare cases the $\mathrm{Ca}$ II triplet). This is far from an ideal spectral region, however. Clearly the $\mathrm{Ca}$ II lines are the best lines to search for; they can, however, be severely filled with emission. This can be seen in the spectra of 17 Lep and AX Mon (Figure 1). In both cases emission completely dominates the photospheric absorption (in 17 Lep the absorption is due to the expanding shell and not the $\mathrm{M}$-star photosphere). If other Be binary systems are similar to these two objects, then possibly the $\mathrm{Ca}$ II emission itself could be helpful in detecting Be binary systems. Before we explore this possibility let us turn our attention to a comparison of the four established Be binary stars.

\section{Binary Be Stars}

Four Be stars are known to be binary systems containing a cool, G, K, or M-type component: AX Mon, 17 Lep, HD 218393, and HR 894 (HD 18552). The spectroscopic and photometric properties of these four systems are summarized in Table I.

TABLE I

Binary Be stars

\begin{tabular}{|c|c|c|c|c|}
\hline & 17 Lep & AX Mon & HD 218393 & HR 894 \\
\hline $\begin{array}{l}\text { Spectrum } \\
\text { Secondary } \\
\text { Contribution. }\end{array}$ & B9V + M2III & $\mathrm{B} 0.5+\mathrm{K} 2 \mathrm{II}$ & B3 + K1III & B8V+gG9: \\
\hline $\begin{array}{l}\text { Contribution: } \\
\mathbf{H} \alpha\end{array}$ & $50 \%$ & $50 \%$ & $20 \%$ & $10 \%$ \\
\hline$\lambda 8500 \AA$ & $75 \%$ & $65 \%$ & $40 \%$ & $25 \%$ \\
\hline$V$ Magnitude & 4.9 & 6.8 & & 6.1 \\
\hline HKL Colors ${ }^{\mathrm{a}}$ & $\begin{array}{l}\text { Late-type } \\
\text { star }\end{array}$ & Free-Free & Free-Free & Free-Free \\
\hline $\begin{array}{l}\text { Emission } \\
\text { Spectrum }\end{array}$ & $\begin{array}{l}\text { Variable } \\
\text { shell }\end{array}$ & $\begin{array}{l}\text { Variable } \\
\text { emission and } \\
\text { shell }\end{array}$ & $\begin{array}{l}\text { Variable } \\
\text { emission and } \\
\text { shell }\end{array}$ & $\begin{array}{l}\text { Constant } \\
\text { emission } \\
\text { (shell?) }\end{array}$ \\
\hline Radial Velocity & Orbit $^{b}$ & Orbit $^{c}$ & Variable $^{d}$ & Variable $^{e}$ \\
\hline
\end{tabular}

a Allen (1973).

b Cowley (1968).

c Cowley (1964).

d Doazan and Peton (1970), Kř́iž and Harmanec (1975).

e Plaskett et al. (1920). 


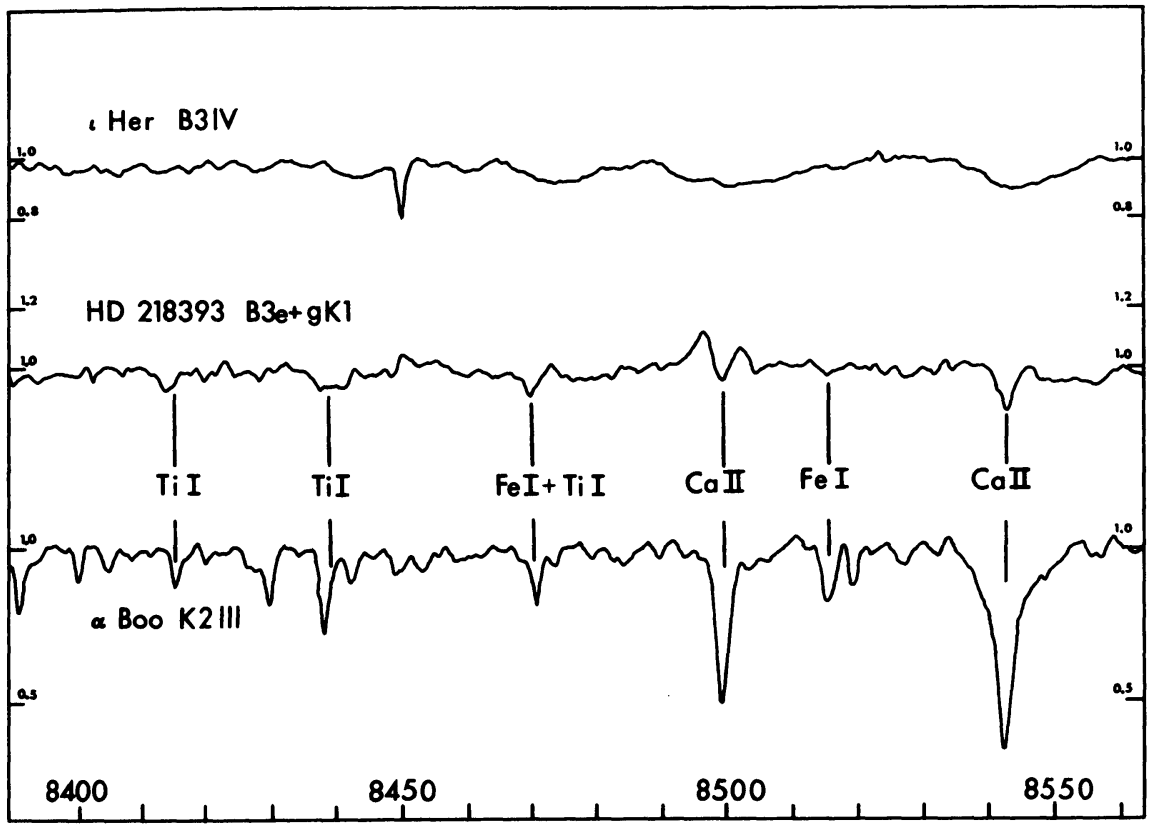

Fig. 2. The infrared spectrum of HD 218393 compared with that of $\iota \mathrm{Her}$ (B3IV) and $\alpha$ Boo (K2III). The most prominent lines of the companion are marked. Ordinate and original dispersion the same as for Figure 1.

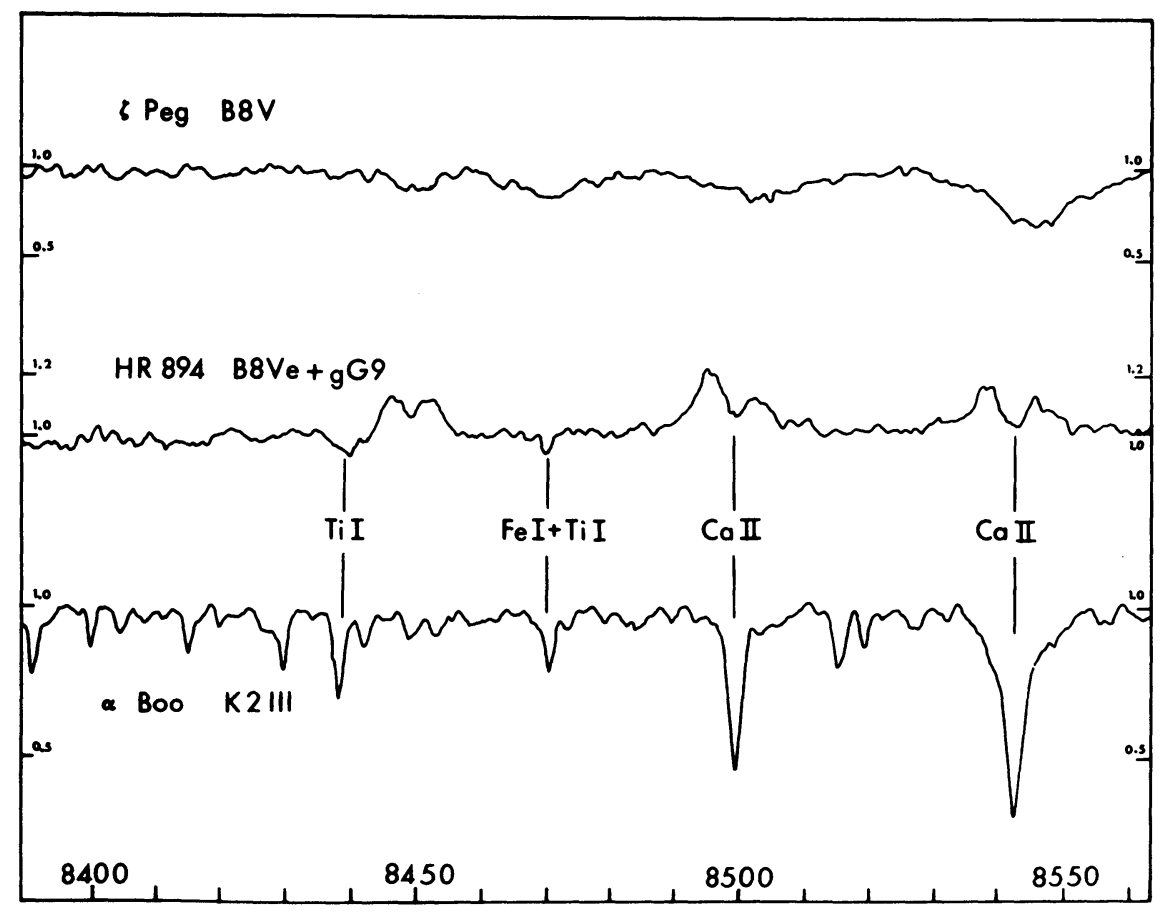

Fig. 3. The infrared spectrum of HR 894 compared with that of $\zeta$ Peg (B8V) and $\alpha$ Boo (K2III). The most prominent lines of the companion are marked. Ordinate and original dispersion the same as in Figure 1. 
The near infrared spectra of AX Mon and 17 Lep are dominated by the cool component of the system (Figure 1). In both systems strong emission is observed at the calcium triplet. Radial velocity measurements of these lines indicate that the emission is associated with the cool component and not with the emission envelope. In 17 Lep strong shell lines severely cut into the calcium emission. The shell lines do not follow the orbital motion of the M-type star but rather remain at the velocity of the expanding shell that surrounds the whole system. Except for these anomalies both companions appear quite normal. The two newer discoveries, HD 218393 (Figure 2) and HR 894 (Figure 3), have considerably less dominating companions. Here, too, both systems display emission at the calcium triplet. It does not, however, dominate the photospheric absorption of the secondary. Very preliminary radial velocity measurements indicate that in both systems the emission may remain at a fixed velocity, the systemic velocity. HR 894 represents the probable limit of detectability for our equipment. The companion is visible only at the calcium triplet and the strongest iron and titanium lines.

Only one common characteristic is found in the infrared spectra of these Be binary stars: emission at the calcium triplet.

\section{Infrared Ca II Emission}

As part of a general spectroscopic investigation of Be stars and related objects in the near infrared over 350 spectrograms in the region of the infrared calcium triplet have been obtained. In total over 120 different objects have been observed. The full program and a description of the equipment used was reviewed earlier in this Symposium. A dispersion of $23 \AA \mathrm{mm}^{-1}$ was used for most objects. All spectra were widened to at least $0.6 \mathrm{~mm}$.

The results of this investigation show that approximately $20 \%$ of all early type emission line stars have calcium triplet emission. If one restricts the observations to only what would be termed 'classical' Be stars only $15 \%$ show calcium triplet emission. Table II lists the stars for which we find emission at the calcium triplet and the strength of the emission. Figure 4 shows the region of the first two calcium lines in three representative stars.

The infrared triplet of calcium $(\lambda \lambda 8498,8542$, and $8662 \AA)$ is severely blended with Paschen lines P16, P15, and P13. This blending has caused earlier investigators to overestimate the frequency of occurrence of calcium triplet emission in Be stars. On lower dispersion plates, such as our $33 \AA \mathrm{mm}^{-1}$ plates and those of earlier investigators, weak $\mathrm{Ca}$ II emission appears to be present in stars showing either a strong Paschen shell spectrum or strong Paschen emission. However, higher dispersion plates taken at the same time do not confirm the presence of the calcium emission, they show only Paschen emission.

The presence or strength of infrared calcium triplet emission is found not to correlate with any obvious parameter of the underlying star or the emission envelope. All B-type spectral sub-classes are equally represented. All types of emission envelopes are represented: strong emission (HD 7636), weak emission (HR 894), early-type shell ( $\phi$ Per), and late-type shell (HD 193182). All degrees of 
TABLE II

Infrared $\mathrm{Ca}$ II emission stars

\begin{tabular}{lll}
\hline Object & Sa II & Comments \\
\hline Continuum & \\
\hline
\end{tabular}

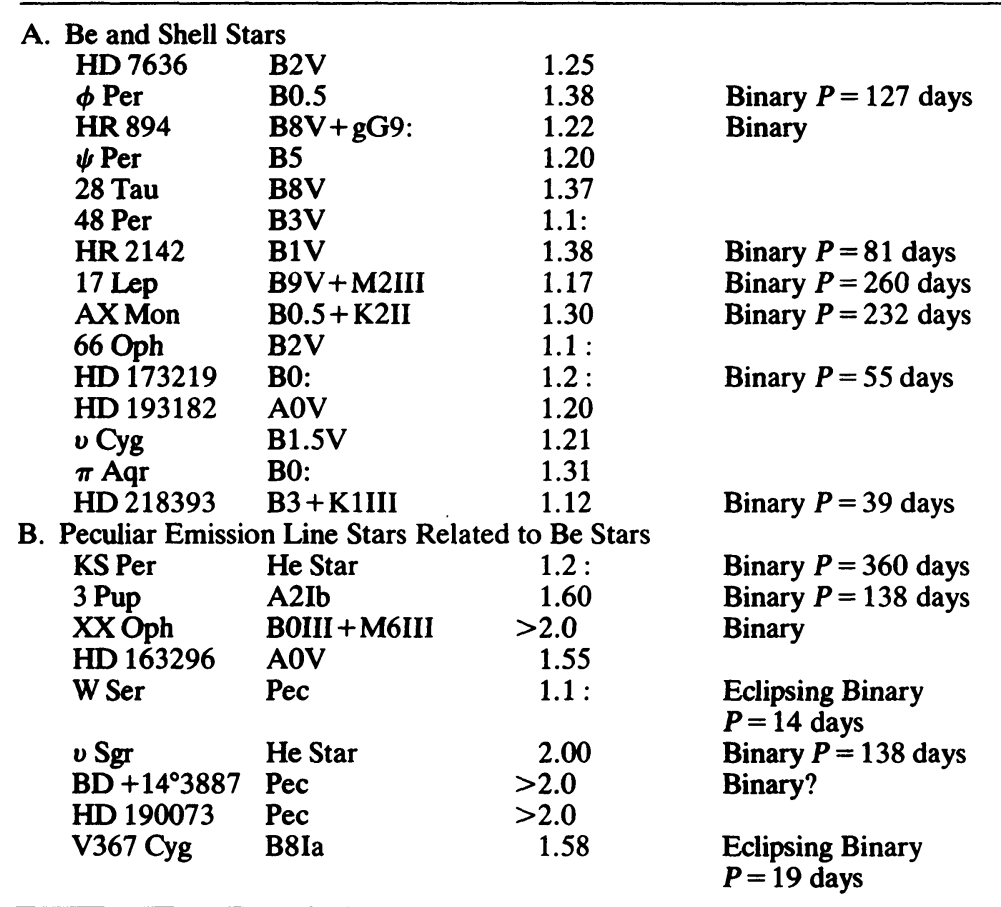

activity are represented, from the very stable (HD 193182) to the very active $(\pi$ Aqr). A wide variety of rotational velocities are also represented. No correlation was found with any photometric property of the star. Color excesses range from $0^{m} .40$ to essentially zero. The far infrared flux distribution typically shows only the expected free-free emission. Here, again, the range is from little or no excess to a reasonably large excess. Some of the peculiar emission line objects show evidence of extensive dust shells; most, however, do not.

\subsection{LINE PROFILES AND STRENGTHS}

The calcium triplet line strengths in classical Be stars range in peak intensity from approximately $10 \%$ above the local continuum to almost $40 \%$ above the local continuum. In the more peculiar Be stars peak intensities in excess of twice the local continuum are not uncommon. The line widths are also quite large: half widths smaller than $350 \mathrm{~km} \mathrm{~s}^{-1}$ are rare. The calcium triplet line widths also do not correlate either with the widths of other emission lines in the spectrum or the $v \sin i$ of the underlying star. 


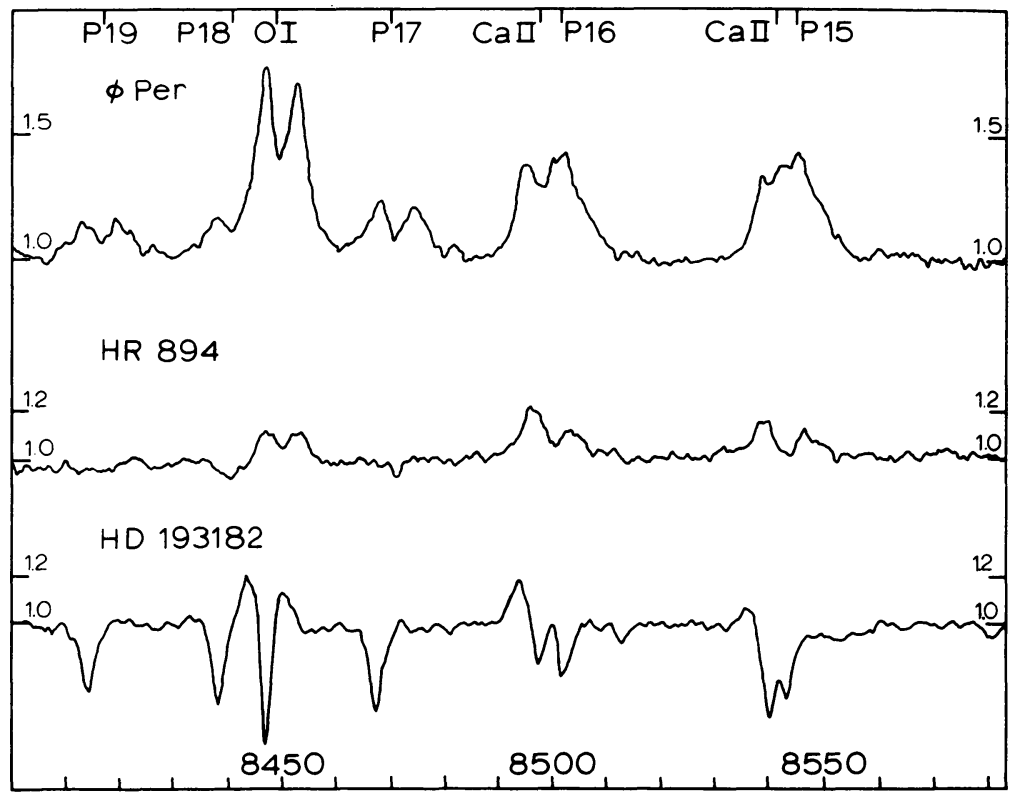

Fig. 4. The region of the first two calcium lines in three calcium triplet emission stars. The shell star HD 193182 also has a strong Ca II shell spectrum. Ordinate and original dispersion the same as for Figure 1.

The expected relative line strengths for the triplet lines are $1: 9: 5$. This is not observed in any case; all show an essentially $1: 1: 1$ intensity ratio. The atomic parameters for these transitions are sufficiently well known that the observed difference cannot be attributed to uncertain physics. The explanation for this anomaly appears to be that the calcium triplet emission in Be stars is very optically thick. This is further supported by the observation that after the removal of Paschen contamination most stars, in particular the classical Be stars, show strong reversals at the line centers; in many the reversal drops very nearly to the continuum. In no case has the reversal been observed to extend below the continuum. Line widths are found to correlate with the strength of the central reversal as expected from optical depth effects. Thus, the inevitable conclusion is that in Be stars the infrared calcium triplet emission arises from a region of large optical thickness.

\subsection{Ca II TRIPLET AND THE H AND K LINES}

The large optical thickness of the $\mathrm{Ca}$ II infrared triplet is difficult to explain in the context of the classical model of a Be star. This problem, however, is surpassed by an even larger problem. Figure 5 shows a partial Grotrian diagram for $\mathrm{Ca}$ II. The lines of the infrared triplet arise from the same upper levels as do the $\mathrm{H}$ and $\mathrm{K}$ lines of calcium. The ratios of the transition probabilities are well known and strongly in favor of the $\mathrm{H}$ and $\mathrm{K}$ lines. However, since we have optically thick lines the relative intensities will go as the relative $B_{\nu}$ 's and not the relative transition probabilities. 


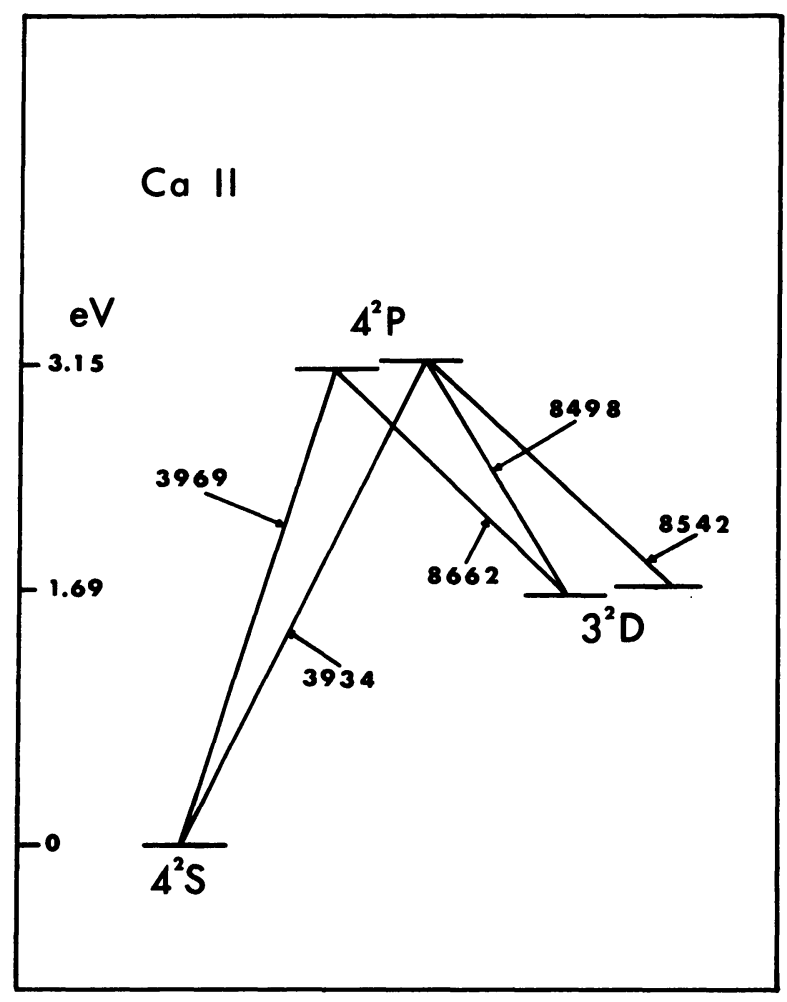

Fig. 5. Partial Grotrian diagram for Ca II.

The line strengths are compared to the local continuum so allowance must be made for the stronger continuum in the region of the $\mathrm{H}$ and $\mathrm{K}$ lines. If this is done then the predicted emission line strengths for the $\mathrm{H}$ and $\mathrm{K}$ lines in the classical Be stars range from 5 to $20 \%$ above the local ( $\mathrm{H}$ and $\mathrm{K}$ ) continuum, assuming typical Be envelope temperatures $(10000-15000 \mathrm{~K})$. However, in all cases $N O$ significant $\mathrm{H}$ and $\mathrm{K}$ emission is observed. No anomaly of any kind, emission or absorption, is observed at the $\mathrm{H}$ and $\mathrm{K}$ lines of the $\mathrm{Be}$ stars showing calcium triplet emission. Photospheric absorption, interstellar gas, and in some cases (shell stars) circumstellar gas can account completely for the violet lines of calcium. Figure 6 illustrates the $\mathrm{K}$ line and the $\lambda 8498 \AA$ infrared line (both arise from the $4 p^{2} P_{3 / 2}$ level) in three classical Be stars and one peculiar emission star (HD 163296).

\subsection{VARIABILITY}

The question of the permanence of calcium triplet emission must now be discussed. This is not easily accomplished, as investigations of Be stars in this region are quite rare. A comparison of our data with the published spectral prints of the previous investigators shows only that major changes have not occurred, except in the case of $\kappa$ Dra, which will be discussed later. Houziaux (1962) has published an intensity 


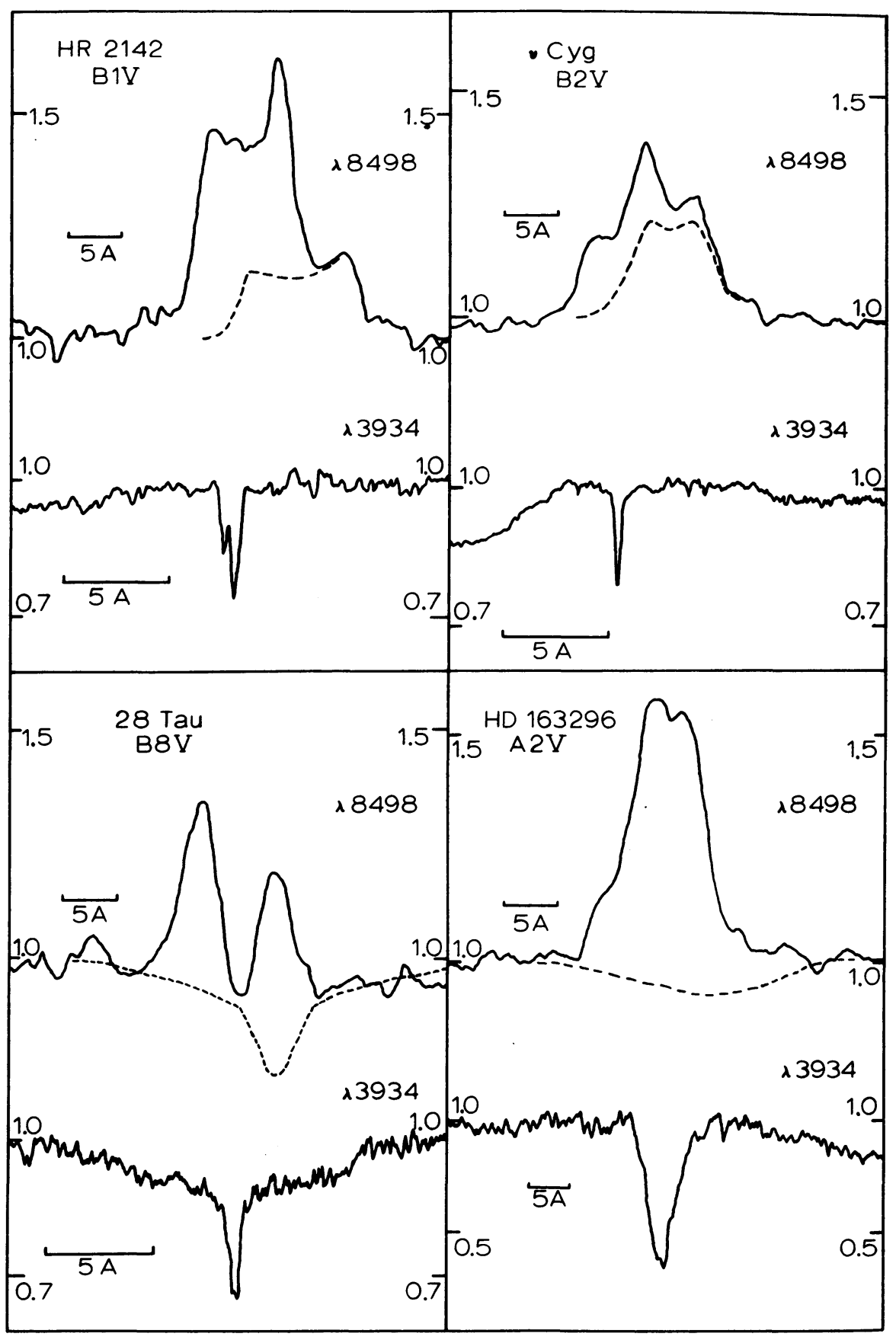

Fig. 6. Comparison of the $\lambda 8498 \AA$ infrared line to the $\lambda 3934 \AA(K)$ line in four calcium emission stars. Ordinate is in units of intensity normalized to the continuum. The profile of P16 is indicated by the dashed line. 
tracing of 28 Tau during one of the star's strong shell phases. We have observed this star during the past three years while its shell has strengthened, declined, and then began to strengthen again. If a comparison is made between our spectra and Houziaux's 1962 spectrum, after removal of the very strong Paschen and calcium shell lines, then one finds that NO significant change occurred in the calcium emission lines. Our observations of three other stars, $\phi$ Per, HR 2142, and HD 163296, have also shown that the calcium emission does not participate in the variations seen in the hydrogen envelope. $\kappa$ Dra is the only Be star to show a definite variation of infrared calcium emission strength. Hiltner (1947) reported strong calcium emission, apparently optically thick, on his spectrogram along with Paschen and $O_{1} \lambda 8446 \AA$ emission. Andrillat and Houziaux (1967) reported that their spectrum shows calcium triplet emission, also apparently optically thick, and $\mathrm{O}_{1}$ $\lambda 8446 \AA$, but Paschen lines in absorption. Our spectra for $\kappa$ Dra are quite similar to that of Andrillat and Houziaux except for one thing: the calcium triplet emission has vanished. This, then, is a case of a major change occurring in the calcium triplet without an accompanying change in the hydrogen envelope.

This lack of correspondence between the emission lines of hydrogen and the emission lines of calcium strongly suggests that they arise in two completely separate regions. This suspicion is further supported when one looks at the mechanisms for the formation of the infrared calcium triplet.

\subsection{Models}

A model for the calcium triplet emission in Be stars must satisfy a number of conditions. The emitting region must be optically thick to all three calcium lines. The predicted $\mathrm{H}$ and $\mathrm{K}$ emission must be sufficiently small as to be undetectable. No enhancement of $\mathrm{H}$ and $\mathrm{K}$ or triplet absorption in the spectrum should be predicted. Reversals in the triplet lines should not drop below the local continuum. The calcium triplet emitting region should not participate in the variations seen in the rest of the envelope. Finally, the calcium region should not give rise to a strong visual or infrared continuum or to any other strong emission lines.

Using simple calculations, an estimate of the temperature of the emitting region can be obtained. Wellman (1951) (see also Pagel, 1960) developed a method for obtaining the volume emission measure, $V N_{e} N_{\text {ion }}$, for an optically thin gas. This method utilizes the total equivalent width of the emission line and assumes that the dominant mechanism of line formation is recombination. If the gas giving rise to the calcium triplet has a temperature typical of that of the hydrogen envelope, 10000 to $15000 \mathrm{~K}$, then recombination will be the dominant line formation mechanism. Remember, however, that the triplet lines are quite optically thick. Wellman's method then can only give an estimate of the lower limit to the size of the emitting region. When applied to the weakest of the triplet lines ( $\lambda 8498 \AA$ ) this lower limit is in itself quite revealing. Very large emitting regions are required: in some cases a total mass of the envelope in excess of $10^{-3}$ solar masses is needed to explain the calcium line strengths. This method also predicts $\mathrm{H}$ and $\mathrm{K}$ emission much stronger than that allowed by the observations. Recombination processes in a hot gas appear unable to even approximately explain the observations. 
A reduction of the gas temperature below $10000 \mathrm{~K}$, specifically to the 5000 $6000 \mathrm{~K}$ range, will alleviate both the problem of the line strengths and the absence of strong $\mathrm{H}$ and $\mathrm{K}$ emission. The calcium triplet has its largest emission per unit volume in this temperature regime. Also, since the line intensities will go as the relative $B_{\nu}$ 's, this temperature regime will strongly favor the triplet lines over the $\mathrm{H}$ and $\mathrm{K}$ lines. This reduction of $\mathbf{H}$ and $\mathbf{K}$ emission, however, is still insufficient to explain the complete absence of emission at $\mathrm{H}$ and $\mathrm{K}$. A further mechanism is required to reduce the $\mathrm{H}$ and $\mathrm{K}$ strength to below the limit of detectability.

The first attempt to identify the cause of this reversal was made by Wyse (1941). Merrill (1934) had observed infrared calcium emission in long-period variables when the $\mathrm{H}$ and $\mathrm{K}$ lines showed only absorption. Wyse suggested that selective photoionizations by $L \alpha$ photons, or in the case of the Be stars the far ultraviolet continuum shortward of $L \alpha$, depopulated the lower ${ }^{2} D$ level thus causing the intensity reversal. Hiltner (1947) found that this explanation could not be reconciled with his observations. With this we completely agree. Our observations are incompatible with Wyse's model. The complete lack of correlation of either the presence or the strength of calcium emission with any attribute of the underlying star or the ionized hydrogen envelope strongly suggests that selective photoionization cannot explain the observed results. It no doubt plays an important role in the line formation process, but it cannot be the sole cause of the intensity reversal.

Herbig (1952) and Kraft (1957) suggested an alternative mechanism to explain the reversal in long-period variables that may have applicability to Be stars. They pointed out that self-absorption by cooler layers of gas outside the emitting region can explain the reversal of intensities. The $\mathrm{H}$ and $\mathrm{K}$ lines will be strongly affected by the cooler gas, whereas the triplet lines will, because of their much lower opacity $\left(f_{3934} / f_{8498} \simeq 75\right)$, pass through the gas essentially unaffected. If indeed in Be stars the calcium emission arises in a region of gas temperature $5000-6000 \mathrm{~K}$, it would not be surprising to find a small amount of cooler $(T \sim 4000 \mathrm{~K})$ gas. Optical depths to the $\mathrm{H}$ and $\mathrm{K}$ lines in this region would need to be in the range $\tau \simeq 1-2$ to reduce the $\mathrm{H}$ and $\mathrm{K}$ emission below the limit of detectability.

If this model is correct for those Be stars displaying calcium triplet emission, then it requires the existence of a two-component envelope: a hot, $T \sim 10000 \mathrm{~K}$, ionized hydrogen disk plus a cool $(T \sim 5000 \mathrm{~K})$ region that shows little variation and does not lie in our line of sight to the Be star. How this not insignificant amount of unionized gas can be maintained in the presence of a hot B-star is as yet not understood.

Throughout this discussion it has been stressed that the presence of calcium triplet emission does not correlate with any attribute of the Be star. This is not entirely true: calcium triplet emission appears to correlate with binary nature. Over one-half of the stars listed in Table II are confirmed binary stars or are strongly suspected of being binary stars. Of the Be stars in our survey not displaying calcium triplet emission less than $10 \%$ are suspected of being binary stars. How the presence of a cool companion causes calcium triplet emission is not completely understood. In some cases (e.g. AX Mon) the emission appears to come from a 'chromosphere' associated with the late-type companion. In other cases (e.g. HD 218393) it appears that the emission may arise in an extensive disk surrounding the entire system, possibly being fed by the cool companion. 
In the case of some of the peculiar stars (e.g. HD 163296) it is possible that the calcium emission is associated with an extensive dust cloud. The stars in Table II for which evidence has been found suggesting that they have large dust shells associated with them (roughly half the peculiar objects and none of the Be stars of Table II) have very different calcium emission lines than the non-dust shell stars. The lines are still optically thick, i.e. the intensity ratio is still $1: 1: 1$, but no line reversals are observed. All lines appear to have simple, or only slightly distorted, Gaussian profiles. This association of strong calcium emission and dust does not appear to apply to all stars. Swings (1973) has observed the star HD 45677 in the region of the calcium triplet and finds no trace of emission. Another parameter must be invoked to explain why some objects with strong infrared excesses have calcium triplet emission and others do not.

\subsection{Conclusions}

Clearly the explanation, or explanations, of the calcium triplet in emission line stars is not yet at hand. The evidence in favor of the source of the emission being relatively cool gas is strong. The origin of the gas and the mechanism by which it is maintained are unknown. The high incidence of interacting binary stars among those stars displaying calcium triplet emission strongly suggests a connection between the two phenomena. The answer to this possible connection lies in further analysis. In particular, a detailed line transfer investigation of the calcium triplet emission lines should supply a set of physical parameters for the emitting region that will allow establishment of unique models for these Be stars.

The one conclusion that is now, I believe, quite obvious is that the $15 \mathrm{Be}$ and shell stars listed in Table II, many of which have been discussed at this Symposium, can no longer be considered 'classical' Be stars in the sense that they cannot be represented by the simple Struve model of a rotationally unstable Be star.

\section{Summary}

Unfortunately the conclusion reached regarding the detectability of cool companions to Be stars was not the one desired. Photometric detection appears to be ruled out for all but the most extreme cases. Spectroscopic detection, still the most promising way to detect companions, is also unlikely to yield many new systems. The problem is quite analogous to that of the longer period Algol systems, where, if it were not for the eclipses little evidence would exist for their binary nature. The existence of infrared calcium triplet emission in a number of $\mathrm{Be}$ stars is quite intriguing. Possibly it is the signature of an interacting binary Be star; certainly it is the indicator of a very unusual Be star.

\section{Acknowledgements}

I wish to thank Dr G. J. Peters and Dr M. Plavec for many helpful discussions on all topics covered in this paper. I would also like to acknowledge their assistance and 
that of Mr E. A. Harlan of Lick Observatory in obtaining many of the observations. I also wish to thank $\mathrm{Dr}$ J. L. Linsky for helpful discussions regarding the calcium triplet paradox. This research was supported by NSF grant MPS 74-04194A01 (Popper/Plavec) as part of a general project studying interacting binary stars.

\section{References}

Allen, D. A.: 1973, Monthly Notices Roy. Astron, Soc. 161, 145.

Andrillat, Y. and Houziaux, L.: 1967, J. Observ. 50, 107.

Cowley, A. P.: 1964, Astrophys. J. 139, 817.

Cowley, A. P.: 1967, Astrophys. J. 147, 609.

Doazan, V. and Peton, A.: 1970, Astron. Astophys. 9, 245.

Gehrz, R. D., Hackwell, J. A., and Jones, T. W.: 1975, Astrophys. J. 191, 675.

Herbig, G. H.: 1952, Astrophys. J..116, 369.

Hill, G. and Hutchings, J. B.: 1970, Astrophys. J. 162, 265.

Hiltner, W. A.: 1947, Astrophys. J. 105, 212.

Houziaux, L.: 1962, Publ. Astron. Soc. Pacific 74, 250.

Johnson, H. L., Mitchell, R. I., Iriarte, B., and Wiśniewski, W. Z.: 1966, Comm. Lunar Planetary Laboratory 4, 99.

Kraft, R. P.: 1957, Astrophys. J. 125, 336.

Kríz, S. and Harmanec, P.: 1975, Bull. Astron. Inst. Czech. $26,65$.

Lockwood, G. W., Dyck, H. M., and Ridgway, S. T.: 1975, Astrophys. J. 195, 385.

Merrill, P. W.: 1934, Astrophys. J. 79, 183.

Pagel, B. E. J.: 1960, Vistas in Astronomy 3, 203.

Peters, G. J.: 1972, Publ. Astron. Soc. Pacific 84, 498.

Peton, A.: 1974, Astrophys. Space Sci. 30, 481.

Plaskett, J. S., Harper, W. E., Young, R. K., and Plaskett, H. H.: 1920, Publ. Dominion Astrophys. Obs. 1, 163.

Slettebak, A.: 1950, Astrophys. J. 112, 559.

Swings, J. P.: 1973, Astron. Astrophys. 26, 443.

Wellman, P.: 1951, Z. Astrophys. 30, 71.

Wyse, A. B.: 1941, Publ. Astron. Soc. Pacific 53, 184.

\section{DISCUSSION}

Meisel: One wonders how much the outer edge of an optically thick shell can look like a K-type star. $\mathrm{K}$-type components might be expected to show the molecular $\mathrm{CN}$ features in the infrared if they are indeed photospheric. I presume that these have not yet been searched for in the infrared spectrum of infrared excess objects.

Polidan: I assume that you are referring to the feature at $\lambda 9150 \AA$. I had hoped to observe it but it is beyond the reach of our Varo tube. There are a few weak $C N$ lines in the water band around $\lambda 8300 \AA$. I am now trying to map out this band and to search for the $\mathrm{CN}$ lines. In some of the stars I think I do see them.

Swings: The observers at Asiago have observed CN absorption bands in two stars, but I do not know if they are on your list.

Snijders: Two fortunate coincidences occur in the $\mathrm{Ca}$ II ion level structure. Firstly the strongest upward transitions from the $4 P$ level are the $4 P \rightarrow 4 D$ lines at about $\lambda 3700 \AA$. Plates obtained for the $H$ and $K$ lines will often cover this region also. Study of the $4 P-4 D$ multiplet will be helpful for establishing both the $4 P$ populations and the importance of recombination processes. Secondly the forbidden $3 D \rightarrow 4 S$ lines are just like the $4 P \rightarrow 3 D$ triplet in the near infrared. Study of the Ca II lines in Be stars would be much easier if observers systematically would give data for all four multiplets, not just the two you just discussed.

Polidan: We have looked for the forbidden $3 D-4 S$ transition in all the triplet emission stars. Only in one case do we find emission, and this is the star $v$ Sgr. Greenstein and Merrill reported the same observation in 1946. 